\title{
Initial conditions in chaotic inflation with modified gravity
}

\author{
Dmitry Gorbunov ${ }^{1, a}$ \\ ${ }^{1}$ Institute for Nuclear Research of Russian Academy of Sciences, 117312 Moscow, Russia
}

\begin{abstract}
Evolution in chaotic inflationary models begins at a high energy density with semi-classical initial conditions presumably consistent with a universal quantum nature of all the fundamental interactions: each quantum contributes the same amount to the energy density. There is an upper limit on this amount inherent in the approach, ensuring that all the quanta are inside the weak-coupling domain. This issue is investigated in realistic models of modified gravity, R2- and Higgs-inflations, with emphasize on the specific change of the initial conditions with metric frame, while all the quanta still contribute equal parts. This illustrates that inflationary models with flat potential may be natural.
\end{abstract}

\section{Introduction}

The Standard Model of particle physics (SM) and General Relativity (GR) fail to describe the cosmology. The major phenomenological problems here are numerous dark matter phenomena and the matter-antimatter asymmetry of the Universe. There are also several puzzling coincidences between cosmological parameters, among them the widely recognized is an order-of-magnitude equality of baryon, dark matter and dark energy contributions to the present energy density of the Universe. The vanilla $\Lambda \mathrm{CDM}$ cosmological model predicts too large number of dwarf galaxies and has other issues probably related to evolution of the matter perturbations at small scales.

There is a separate class of problems called the problem of initial conditions for the Hot Big Bang Theory. Since the homogeneous Universe may form spherical or hyperbolic 3-dimensional manifold, its flatness at present is mysterious. Both at radiation and at matter dominated stages of the Universe expansion the size of causally connected region, particle horizon, grows faster than a physical spatial size does, so the Universe must be highly inhomogeneous at large scales, that is quite contrary to all observations. The Universe expansion is adiabatic, keeps the entropy conserved in a comoving volume, hence the entropy density in the very early Universe was enormously high $s \ggg M_{\mathrm{Pl}}^{3}$, which is weird. Both radiation and matter dominated stages imply singular energy density at the very beginning of expansion, $\rho \propto 1 / t^{2}$. If SM is a remnant of some Grand Unified Theory, the corresponding phase transition(s) at the Hot stage generally populates the Universe with topological defects; later some of these relics, in particular monopoles and domain walls, become dominant and overclose the Universe.

The initial condition problems can be elegantly solved by introducing an (almost exponentially) fast expansion preceding the Hot stage. Then the curvature (if any) grows exponentially making the Universe exponentially flat. An originally causally connected region, whatever tiny, becomes exponentially large and today we actually observe only its minuscule part. Any heavy stable relics

a e-mail: gorby@ms2.inr.ac.ru 
(like monopoles) are at exponentially large distances one from each other, so the late Hot Universe is safe. There are no initial singularities in the exponentially expanding Universe.

This stage, called inflation, can be naturally organized by a slowly evolving homogeneous scalar field $\phi$, inflaton, see Fig. 1. One of the most popular idea here is chaotic inflation [1].

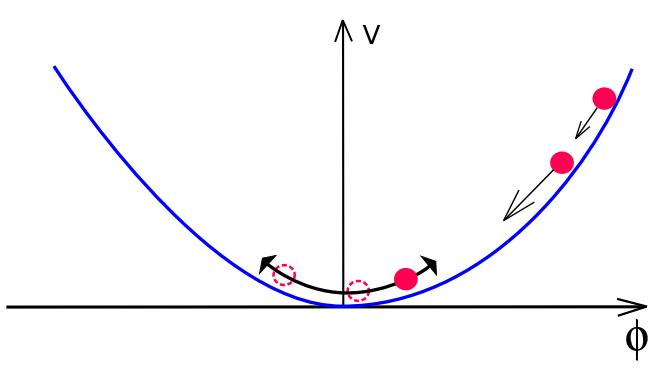

Figure 1. In a model of chaotic inflation with large field [1] the inflaton slowly rolls along the potential slope mimicking almost constant energy density, so the Universe exhibits (almost) exponential expansion. The slow rolling stops at some point and the inflaton starts to oscillate, so the Universe enters a stage of decelerating expansion. The hot stage starts after reheating, when the inflaton transfers its energy density into ultrarelativistic (SM) particles.

\section{Chaotic inflation and unlikeness of models with flat potential}

In the chaotic inflation field $\phi$ rolls down along the potential $V(\phi)$ starting from large, generally transplanckian values, $\phi>M_{\mathrm{Pl}}$, see Fig. 1 . In the Robertson-Walker metric, $d s^{2}=d t^{2}-a^{2}(t) \mathbf{d x} \mathbf{x}^{2}$, with $a(t)$ being the scale factor, $\phi(t, \mathbf{x})$ solves equation

$$
\ddot{\phi}-\frac{\Delta \phi}{a^{2}}+3 \mathcal{H} \dot{\phi}+V^{\prime}(\phi)=0 .
$$

The Hubble parameter $\mathcal{H} \equiv \dot{a} / a$ obeys the Friedmann equation

$$
\mathcal{H}^{2}=\frac{8 \pi}{3 M_{\mathrm{Pl}}^{2}}\left(\frac{1}{2} \dot{\phi}^{2}+\frac{1}{2 a^{2}}(\nabla \phi)^{2}+V(\phi)\right)
$$

and plays a role of dynamical friction in Eq. (1). Actually, if the field evolves very slowly, then its energy density is dominated by the scalar potential $V(\phi)$ and the Hubble parameter remains almost constant, see Eq. (2), ensuring the almost exponential expansion, $a(t) \propto \exp (\mathcal{H} t)$. Then the gradient terms in Eqs. (1), (2), being suppressed by the growing scale factor, get nullified and the Hubble friction governs the field homogeneous slow rolling towards the origin of scalar potential, $\phi=\phi(t) \rightarrow$ 0 . The kinetic terms in Eqs. (1), (2) are negligible if the so-called slow roll parameters are small,

$$
\frac{M_{\mathrm{P}}^{2}}{2}\left(\frac{V^{\prime}}{V}\right)^{2} \equiv \epsilon \ll 1, \quad M_{\mathrm{P}}^{2} \frac{V^{\prime \prime}}{V} \equiv \eta \ll 1 .
$$

where we introduce the reduced Planck mass as $M_{\mathrm{P}} \equiv M_{\mathrm{Pl}} / \sqrt{8 \pi}$. One can check that the latter conditions are satisfied for any power-law scalar potential, $V(\phi) \propto \phi^{\alpha}$, if the scalar field takes a transplanckian value, $\phi_{i}>M_{\mathrm{Pl}}$. Then the field goes down slowly and, once $\epsilon \simeq 1$ or $\eta \simeq 1$, the inflation terminates. 


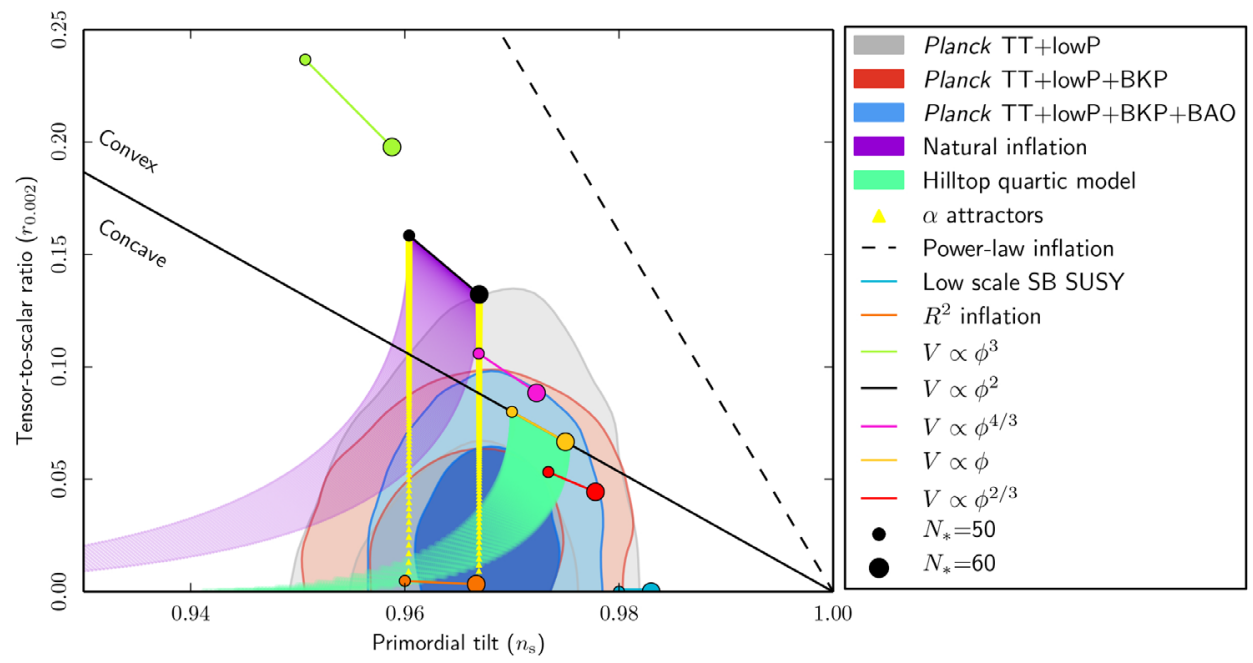

Figure 2. Cosmological constraints [4] on inflationary models. The colored regions are allowed at $95 \% \mathrm{CL}$, there are predictions of various cosmological models.

The chaotic initial conditions for the inflaton field imply [1,2] that the scalar field fluctuates in any Planck-size region, and all the contributions to the energy density -kinetic term, gradient term and potential term- are (on average) of the same order, which is Planckian, i.e.

$$
\frac{1}{2} \dot{\phi}^{2} \sim \frac{1}{2}\left(\partial_{i} \phi\right)^{2} \sim V(\phi) \sim M_{\mathrm{Pl}}^{4}
$$

Then if in a particular Planck-size patch the potential term becomes somewhat dominant by chance, as a result of the fluctuations, the dynamics of the scalar field (1) rapidly approaches the slow roll and the chaotic inflation begins in this spatial region.

Inflation itself is almost untestable: the only exception is the prediction of exponentially flat Universe, that is in agreement with observations at present. However, it is a general prediction, inherent in the very paradigm of inflation. To recognize a particular inflationary model we need something else. Quite remarkably, the inflation provides with an appropriate feature. Namely, inflaton inhomogeneities $\delta \phi(t, \mathbf{x})$ (linear perturbations about the homogeneous inflationary solution) originated from the inflaton quantum fluctuations can seed the matter inhomogeneities (dubbed scalar perturbations) in the late Universe. Their subsequent evolution initiates large scale structure formation and explain the anisotropy of cosmic microwave background (CMB) radiation $\delta T / T \sim 10^{-4}$.

The scalar perturbation spectrum is almost flat. Both its amplitude and small tilt depend on the inflationary model parameters, and a combined analysis of sensitive to matter inhomogeneities cosmological observables allows one to discriminate between various inflationary models, for details see e.g. Ref. [3]. Figure 2 shows that the present data tend to favor the large field inflation models with concave scalar potential, e.g. the power-law potential $V(\phi) \propto \phi^{\alpha}$ with $\alpha<1$. Among them are theoretically well motivated models with asymptotically flat potentials: $R^{2}$-inflation [5], Higgs-inflation [6], moduli inflation in supersymmetric extensions of the SM, etc. To explain the CMB anisotropy 
$\delta T / T \sim 10^{-4}$ the inflaton plateau must be at about

$$
V_{0} \simeq 10^{-12} M_{\mathrm{Pl}}^{4} .
$$

At first glance [7], however, these models seem to be unlikely in the framework of the chaotic inflation. Indeed, at the Planck epoch, when kinetic and gradient terms take the Planckian values according to conditions (4), the potential (being flat) is fixed by the cosmological observations at much lower level (5),

$$
\frac{1}{2} \dot{\phi}^{2} \sim \frac{1}{2}\left(\partial_{i} \phi\right)^{2} \sim M_{\mathrm{Pl}}^{4} \gg V(\phi),
$$

so that slow roll never happens. In such a model the chaotic inflation requires a beginning with all terms of the same order that is much smaller than the Planckian value, which seems unnatural. Below we illustrate the problem with two prominent examples and explain its solution by correctly imposing the chaotic initial conditions, for more details see Refs. [8, 9].

\section{An example: $R^{2}$-inflation}

Let us consider the first well-developed inflationary model [5] based on modified gravity with action

$$
S^{J F}=-\frac{M_{P}^{2}}{2} \int \sqrt{-g} d^{4} x\left(R-\frac{R^{2}}{6 \mu^{2}}\right),
$$

which after the convenient Weyl transformation (from the Jordan frame to the Einstein frame),

$$
g_{\mu v} \rightarrow \tilde{g}_{\mu v}=\Omega^{2} g_{\mu v}, \quad \Omega^{2} \equiv \exp \left(\sqrt{\frac{2}{3}} \frac{\phi}{M_{P}}\right),
$$

casts into the standard Einstein-Hilbert term and terms describing the scalar degree of freedom, dubbed scalaron, emerging in the gravity sector,

$$
S^{E F}=\int \sqrt{-\tilde{g}} d^{4} x\left[-\frac{M_{P}^{2}}{2} \tilde{R}+\frac{1}{2} \tilde{g}^{\mu v} \partial_{\mu} \phi \partial_{\nu} \phi-\frac{3 \mu^{2} M_{P}^{2}}{4}\left(1-\frac{1}{\Omega^{2}(\phi)}\right)^{2}\right] .
$$

The model has an exponentially flat potential (see left panel of Fig. 3), which approaches (5) to be consistent with the observed CMB anisotropy. Naively, within the chaotic paradigm, the model suffers from unlikeness problem (6).

This is not true in fact. Indeed, in the Jordan frame the chaotic initial conditions look like $\left|R^{J F}\right| \sim$ $M_{\mathrm{Pl}}^{2}$, and matching to cosmological data infers

$$
\Omega^{2}=1-\frac{R^{J F}}{3 \mu^{2}} \sim 10^{12} .
$$

The scalar curvature in the Jordan frame is related to that in the Einstein frame and scalaron as follows,

$$
-R^{J F}=-\Omega^{2} R^{E F}+\Omega^{2} \frac{8 \pi}{M_{\mathrm{Pl}}^{2}} g_{\mu \nu}^{E F} \partial^{\mu} \phi \partial^{v} \phi-\Omega^{2} \frac{4 \sqrt{3 \pi}}{M_{\mathrm{Pl}}} g_{\mu \nu}^{E F} \partial^{\mu} \partial^{v} \phi .
$$

The first and second terms in the r.h.s. above refer to the pure gravity and scalaron contributions to the energy density, respectively. Hence they must be of the same order within the paradigm of chaotic inflation. The third term happens to be of the same order provided the equations of motion. 

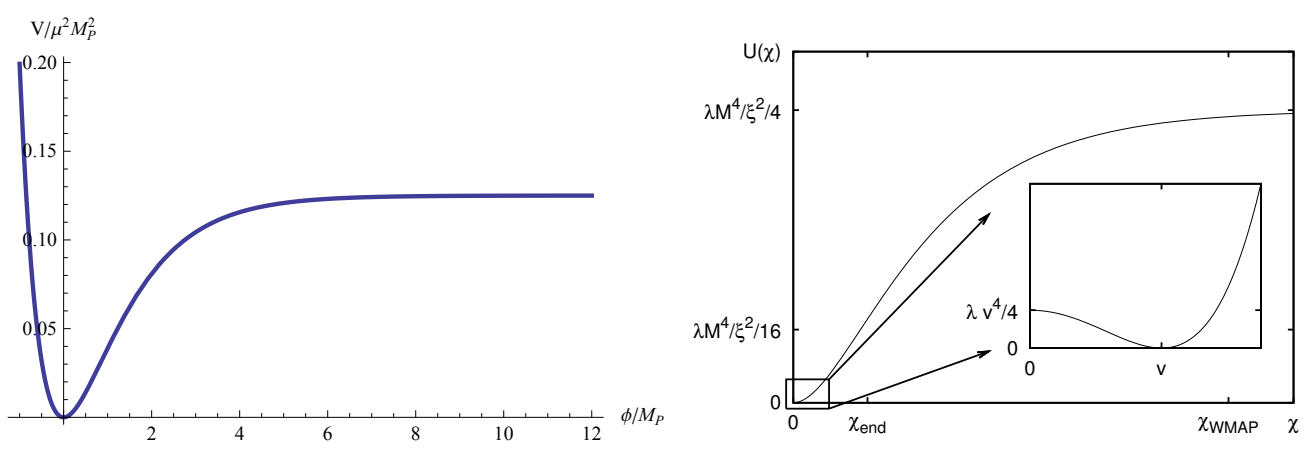

Figure 3. Examples of a flat potential in $R^{2}$-inflation (left panel) and Higgs-inflation (right panel).

Therefore one has

$$
\left|R^{J F}\right| \Omega^{-2} \sim\left|R^{E F}\right| \sim \dot{\phi}^{2} M_{\mathrm{Pl}}^{2} \sim\left(\partial_{i} \phi\right)^{2} M_{\mathrm{Pl}}^{2},
$$

which means that all the terms in the Einstein frame are actually of the same order, consistent with the requirement of successful inflation,

$$
\frac{1}{2} \dot{\phi}^{2} \sim \frac{1}{2}\left(\partial_{i} \phi\right)^{2} \sim V(\phi) \sim 10^{-12} \times M_{\mathrm{Pl}}^{4}
$$

There is no unlikeness problem in the $R^{2}$-inflation.

Moreover, the very idea of starting $R^{2}$-inflation with Einstein-frame initial conditions (6) is ridiculous, since in the Jordan frame, due to the Weyl transformation with scale-factor (8), the curvature then much exceeds the Planckian values, see eq. (9). One simply cannot treat semiclassically the quanta naturally adopted to formulate the initial conditions in the chaotic approach. On the contrary, one can start with all the quanta at the upper border of the weak coupling domain, which in the Jordan frame with lagrangian (7) implies large curvature $R^{J F} \gg \mu^{2}$. Then the border under discussion moves from the Planck scale to

$$
\Lambda^{J F} \simeq \frac{M_{\mathrm{P}}}{\sqrt{6} \mu} \sqrt{R^{J F}}
$$

and the contribution of dominating in (7) $R^{2}$-term to the energy density fluctuations,

$$
E^{J F^{4}} \sim \frac{M_{\mathrm{P}}^{2}}{\mu^{2}} R^{J F^{2}},
$$

is well inside the weak coupling domain, limited from above by the new strong coupling scale $\Lambda^{J F}$, as $E^{J F^{4}} \ll \Lambda^{J F^{4}}$. Note, that the corresponding to $E^{J F}$ energy scale in the Einstein frame does not depend on the value $E^{J F}$,

$$
E^{E F} \sim \Omega^{-1} E^{J F} \sim \frac{M_{\mathrm{P}}}{\Lambda^{J F}} E^{J F} \sim \sqrt{\mu M_{\mathrm{P}}},
$$

where in the Weyl scale-factor (8) we express the curvature $R^{J F}$ through the strong coupling scale $\Lambda^{J F}$. One concludes that in the Einstein frame all the terms contributing to energy density (10) are of the same order $E^{E F^{4}}$, cf. (11), and inside the weak-coupling domain. 


\section{One more example: Higgs inflation}

To cook the successful inflation with the SM Higgs weak doublet $H$ one must introduce its nonminimal coupling to gravity [6],

$$
S=\int d^{4} x \sqrt{-g}\left(-\frac{M_{P}^{2}}{2} R-\xi H^{\dagger} H R+\mathcal{L}_{S M}\right)
$$

or written in the unitary gauge $H^{T}=(0,(h+v) / \sqrt{2})($ neglecting $v=246 \mathrm{GeV})$

$$
S=\int d^{4} x \sqrt{-g}\left(-\frac{M_{P}^{2}+\xi h^{2}}{2} R+\frac{\left(\partial_{\mu} h\right)^{2}}{2}-\frac{\lambda h^{4}}{4}+\mathcal{L}_{S M}\right) .
$$

Performing the Weyl transformation

$$
g_{\mu \nu}=\Omega^{-2} \tilde{g}_{\mu v}, \quad \Omega^{2}=1+\frac{\xi h^{2}}{M_{P}^{2}}
$$

one finds in the Einstein frame for the canonically normalized field $\chi$

$$
\frac{d \chi}{d h}=\frac{M_{P} \sqrt{M_{P}^{2}+(6 \xi+1) \xi h^{2}}}{M_{P}^{2}+\xi h^{2}}
$$

the scalar potential (see right panel of Fig. 3)

$$
U(\chi)=\frac{\lambda M_{P}^{4} h^{4}(\chi)}{4\left(M_{P}^{2}+\xi h^{2}(\chi)\right)^{2}}=\frac{\lambda M_{P}^{4}}{4 \xi^{2}}\left(1-\exp \left(-\frac{\sqrt{2} \chi}{\sqrt{3} M_{P}}\right)\right)^{2}
$$

at relevant for inflation values $h \gg M_{P} / \sqrt{\xi}$. It looks exactly as the scalaron potential in the previous example of $R^{2}$-inflation, ${ }^{1}$ see Fig. 3 , and the correct height of the potential plateau (5) is achieved with [6]

$$
\frac{\xi}{\sqrt{\lambda}} \simeq 5 \times 10^{4}
$$

assuming the Higgs self-coupling is positive at the inflationary scale. Since the two potentials coincide one naively expects [7] here the same unlikeness problem.

Actually, there is no unlikeness problem here. Similarly to the case of $R^{2}$-inflation, one starts with the chaotic initial conditions implemented in the Jordan frame,

$$
\Omega^{2} M_{\mathrm{Pl}}^{2} R^{J F} \sim \xi \dot{h}^{2} \sim \xi\left(\partial_{i} h\right)^{2} \sim \lambda h^{4} \sim M_{\mathrm{Pl}}^{4} .
$$

Here we account for the fact that the nonminimal gravity coupling to the Higgs field effectively changes the Higgs kinetic and gradient terms, and chose the dominant parts. Consequently,

$$
R^{J F} \sim \Omega^{-2} M_{\mathrm{Pl}}^{2},
$$

and since the cosmological observations fix the potential in the Einstein frame at the value (5), hence the Weyl scale-factor is

$$
\Omega^{2} \sim 10^{6} .
$$

\footnotetext{
${ }^{1}$ Predictions of these two models for the scalar and tensor perturbation spectra differ only slightly, see Refs. [10, 11], because of different post-inflationary history which culminates in different reheating temperatures [12, 13].
} 
Then in the Einstein frame one arrives at the same picture: all the terms are of the same order needed for the successful inflation, namely

$$
\frac{1}{2} \dot{\phi}^{2} \sim \frac{1}{2}\left(\partial_{i} \phi\right)^{2} \sim \Omega^{-4} M_{\mathrm{Pl}}^{4} \sim 10^{-12} \times M_{\mathrm{Pl}}^{4} .
$$

There is no unlikeness problem in the Higgs-inflation either.

Analogously to the case of $R^{2}$-inflation one can raise the question of the strong-coupling scale in this model and its relation to the values associated with the chaotic initial conditions. Even for small fields the strong-coupling scale in the model (12) is well below the Planck scale [14], namely it is at $M_{P} \sqrt{\lambda} / \xi \sim 10^{14} \mathrm{GeV}$ given the much exceeding unity value of the non-minimal coupling (13). At large homogeneous fields the non-minimal coupling effectively changes the Planck mass, pushing the scale of strong gravity up to

$$
\Lambda^{J F} \simeq \sqrt{8 \pi \xi} h \sim \Omega M_{\mathrm{Pl}},
$$

and the semiclassical treatment of the gravitational sector is applicable only at energy densities below $\Lambda^{J F^{4}}$. The chaotic initial conditions (14) obey this constraint, moreover, the corresponding energy scale $\sim h$ is right at the upper border of the weak-coupling domain, which is limited by the scalar, rather than gravity, dynamics, see Ref. [14] for details. The Weyl transformation with scale-factor (15) leads to the chaotic initial conditions in the Einstein frame (16), which implies the energy scale $\sim \Omega^{-1} h \sim M_{\mathrm{Pl}} / \sqrt{\xi}$ right at the upper border of the weak-coupling domain in this frame [14]. As in case of the $R^{2}$-inflation this scale does not depend on the value $h$ of strong coupling scale in the Jordan frame. Again, the initial conditions like (6) imposed in the Einstein frame would imply working outside the weak-coupling regime, which is not treatable.

\section{Conclusion: initial conditions in an arbitrary frame}

We find that the chaotic paradigm works perfectly well for both $R^{2}$-inflation and Higgs-inflation: they do not suffer from the unlikeness problem. The presented analysis suggests a simple prescription for implementing the chaotic initial conditions in any model as follows. Start in any frame with all contributions to the energy density being of the same order (say, $\sim M_{\mathrm{Pl}}^{4}$ or any other value). Then in any other frame the corresponding contributions are always of the same (though another) order (say, $\sim 10^{-12} \times M_{\mathrm{Pl}}^{4}$ in the EF). The natural energy scale of the corresponding contributions is argued to be the strong coupling scale in a given frame. This statement is consistent with the Weyl transformation, which matches the energy scales of different frames.

The work is supported by the RSF grant 14-12-01430.

\section{References}

[1] Linde A.D., Phys. Lett. B 129, 177 (1983).

[2] Linde A.D., Rept. Prog. Phys. 47, 925 (1984).

[3] Gorbunov D.S. and Rubakov V.A., Introduction to the theory of the early universe: Cosmological perturbations and inflationary theory, (Hackensack, USA: World Scientific, 2011), 489.

[4] Ade P.A.R. et al. [Planck Collaboration], Astron. Astrophys. 594, A20 (2016).

[5] Starobinsky A.A., Phys. Lett. B 91, 99 (1980).

[6] Bezrukov F.L. and Shaposhnikov M.E., Phys. Lett. B 659, 703 (2008).

[7] Ijjas A., Steinhardt P.J. and Loeb A., Phys. Lett. B 723, 261 (2013). 
[8] Gorbunov D.S. and Panin A.G., Phys. Lett. B 743, 79 (2015).

[9] Gorbunov D. and A. Panin A., "Strong-coupling scale and frame-dependence of the initial conditions for chaotic inflation in models with modified (coupling to) gravity," arXiv:1607.05268 [gr-qc].

[10] Bezrukov F.L. and Gorbunov D.S., Phys. Lett. B 713, 365 (2012).

[11] Gorbunov D. and Tokareva A., JCAP 1312, 021 (2013).

[12] Gorbunov D.S. and Panin A.G., Phys. Lett. B 700, 157 (2011).

[13] Bezrukov F., Gorbunov D. and Shaposhnikov M., JCAP 0906, 029 (2009).

[14] Bezrukov F.L., Magnin A. and Shaposhnikov M., Phys. Lett. B 67588 (2009). 